Article

\title{
Exceptional Experiences of Stable and Unstable Mental States, Understood from a Dual-Aspect Point of View
}

\author{
Harald Atmanspacher ${ }^{1,2, *}$ and Wolfgang Fach ${ }^{2}$ \\ 1 Collegium Helveticum, ETH Zurich and University Zurich, 8092 Zurich, Switzerland \\ 2 Institute for Frontier Areas of Psychology and Mental Health, 79098 Freiburg, Germany; fach@igpp.de \\ * Correspondence: atmanspacher@collegium.ethz.ch
}

Received: 16 January 2019; Accepted: 1 February 2019; Published: 15 February 2019

check for updates

\begin{abstract}
Within a state-space approach endowed with a generalized potential function, mental states can be systematically characterized by their stability against perturbations. This approach yields three major classes of states: (1) asymptotically stable categorial states, (2) marginally stable non-categorial states and (3) unstable acategorial states. The particularly interesting case of states giving rise to exceptional experiences will be elucidated in detail. Their proper classification will be related to Metzinger's account of self-model and world-model, and empirical support for this classification will be surveyed. Eventually, it will be outlined how Metzinger's discussion of intentionality achieves pronounced significance within a dual-aspect framework of thinking.
\end{abstract}

Keywords: acategoriality; state-space approach; mental representation; dual-aspect monism; exceptional experiences; intentionality; mind-matter relations

\section{Introduction}

In this article, we address a few points of Thomas Metzinger's seminal account of self and world as representations [1] and propose some connections to areas of research that he himself did not consider much (so far). One of these areas is a dynamical-systems point of view toward his representational approach. The second is an application of this view to recent research on the phenomenology of so-called exceptional experiences. Our third focus in this essay is to link key features of his representational account with the metaphysical position of dual-aspect monism.

In the following Section 2, we give a terse introduction into one property of dynamical systems that is of fundamental significance for their understanding in general: stability. The study of stability in dynamical systems dates back to the work of Lyapunov, Poincaré and others at the turn of the 20th century. It is the conceptual core of recent work that gained publicity in cognitive neuroscience as a "free-energy principle" [2]. States of a dynamical system (such as the brain, or the mental system) can be stable and they can be unstable, depending on the potential landscape that constrains and governs their evolution.

Section 3 employs the different kinds of stability or instability to characterize mental states and representations. An important point here is the systematic distinction of different kinds of dynamics: states typically evolve on timescales that are short as compared to those of representation changes. As a consequence, one can discuss the time evolution of states "adiabatically", i.e., for a quasi-fixed collection of representations modeled by a potential landscape. This leads to a threefold classification of states (first proposed in [3]): categorial states (actualizing mental representations), non-categorial states (in the absence of representations), and acategorial states (between non-actualized representations).

Section 4 utilizes these concepts of states to address different classes of exceptional experiences (EEs), i.e., experiences that deviate from a subject's ordinary phenomenal model of reality (Metzinger's 
PRM). Within a dual-aspect framework of thinking, where the two aspects resemble Metzinger's distinction of self-model and world-model, we outline four basic classes of phenomena constituting EEs and describe the rich empirical evidence that has been established in support of this classification. Moreover, we indicate how the phenomenal experience of EEs relates to categorial, non-categorial and acategorial mental states.

In Section 5, we address a few conspicuous links between Metzinger's representational account and a particular variant of dual-aspect monism: the Pauli-Jung conjecture [4]. The notion of intentionality, central in Metzinger's work as a technical term to address meaning as a reference relation between elements of the self-model and of the world-model, is used to characterize EEs that relate the mental with the physical. The phenomenal model of the intentionality relation (Metzinger's PMIR) is transparent if the relation is not explicitly experienced as meaningful, and its opacity increases with an increasing intensity of experienced meaning.

Finally, we outline how Metzinger's framework of thinking can be elegantly embedded in a dual-aspect framework with a self-model (roughly) corresponding to the mental aspect and a world-model (roughly) corresponding to the physical aspect. While the former is key to Metzinger's position, the latter is less well articulated in his work and might profit from some clarification.

\section{Stability Properties of Dynamical Systems}

The state $\phi$ of a dynamical system, evolving continuously as a function of time $t$, is characterized by a number $n$ of properties (technically speaking, observables) that are represented as the coordinates of an $n$-dimensional state space. The trajectory of the state $\phi(t)$ in its state space represents the evolution of the system. The stability of dynamical systems under small perturbations or fluctuations can be evaluated by a stability analysis. ${ }^{1}$ Such a stability analysis yields so-called Ljapunov exponents $\lambda_{i}(i=1, \ldots, n)$, quantifying how and to which extent fluctuations vary as time proceeds.

Positive Ljapunov exponents indicate an exponential growth of fluctuations, whereas negative ones indicate that fluctuations are damped exponentially. The sum of all Ljapunov exponents is smaller than zero for dissipative systems and vanishes for conservative systems. In the dissipative case, there exists a subspace of the state space, onto which the trajectory of the system is restricted (after an initial "transient" phase). This subspace is called the attractor of the system. Another (larger) subspace, which covers all those (initial) states asymptotically evolving into the attractor, is called the basin of attraction. For a given attractor, the Ljapunov exponents are invariant with respect to continuous transformations of the coordinates of the state space.

The simplest case of an attractor is a "fixed point" in state space, where all $\lambda_{i}$ are negative. If there is no other attractor in the state space, the entire (admissible) state space is the corresponding basin of attraction. More interesting (and more complicated) are situations in which the sum of all $\lambda_{i}$ is negative, yet individual $\lambda_{i}$ are positive. In this case, one speaks of "strange attractors" or "chaotic attractors". Even though the behavior of such systems is governed by strictly deterministic equations, it appears "chaotic" - which is the origin of the notion of deterministic chaos.

The stability of attractors can be studied qualitatively using a method also introduced by Ljapunov (see [8], chapter 7.6). In order to do so, a function $V(x) \geq 0$ is considered in a neighborhood $G$ of a reference point $x=x_{r}{ }^{2}$ The significance of $V(x)$ is that of a potential, which does not need to be a (physical) energy, whose extremal properties in $G$ determine the stability of a state $\phi$ at $x_{r}$. The first derivative $\nabla V(x)=\frac{d V(x)}{d x}$ of $V(x)$ with respect to $x$ describes the change of $V(x)$ in $G$, and the second derivative $\nabla^{2} V(x)=\frac{d V^{2}(x)}{d x^{2}}$ indicates a local maximum $\left(\nabla^{2} V(x)<0\right)$ or minimum $\left(\nabla^{2} V(x)>0\right)$. Using $V$, the following definitions of stable and unstable states can be formulated.

\footnotetext{
See, e.g., [5,6] or other relevant literature for details of this procedure; see also [7].

2 For the sake of simplicity, we consider $V$ as a function of only one variable $x$. Of course, this can be generalized to any number $n$ of variables.
} 
1. A state $\phi$ at $x_{r}$ is stable if $\nabla^{2} V(x) \geq 0$ in $G$. In this case, $V(x)$ is a Ljapunov function:

(a) A state $\phi$ at $x_{r}$ is marginally stable if $\nabla^{2} V(x)=0$ in $G$.

(b) A state $\phi$ at $x_{r}$ is asymptotically stable if $\nabla^{2} V(x)>0$ in $G$.

2. A state $\phi$ at $x_{r}$ is unstable if $\nabla^{2} V(x)<0$ in $G$.

For the simple example of a fixed point attractor in one dimension, Figure $1 \mathrm{~b}$ illustrates case $1 \mathrm{~b}$ for a quadratic potential $V(x)=\alpha x^{2}, \alpha>0$, which is concave in the neighborhood of the minimum of $V(x)\left(\nabla^{2} V>0\right)$. This minimum represents a fixed point with one negative Ljapunov exponent, and a state at this fixed point is asymptotically stable. In case 1a, the gradient of the potential vanishes, $\nabla V=0$, and the second derivative too, $\nabla^{2} V=0$, so that each point for this potential is marginally stable (Figure 1a), corresponding to a vanishing Ljapunov exponent.

(a)

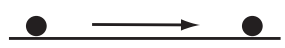

$\mathrm{V}=$ const $\nabla \mathrm{V}=0$

(b)

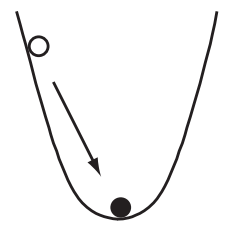

$V=$ concave

$\nabla \mathrm{V} \neq 0$

$\mathrm{V}_{0}=\min$

Figure 1. Kinds of stability of a state: (a) marginal stability for a constant potential $V$ with $\nabla V=0$ and $\nabla^{2} V=0 ;(\mathbf{b})$ asymptotic stability at a critical point $V_{o}$ of a concave potential with $\nabla^{2} V>0$.

A combination of case (2) with case (1b) is illustrated in Figure 2. In the neighborhood of $x_{r}$, at the local maximum, $V$ is convex $\left(\nabla^{2} V<0\right)$, whereas $V$ is concave $\left(\nabla^{2} V>0\right)$ around the potential minima. A state at or around the local maximum is therefore unstable. If the system is in such a state, it will spontaneously relax into one of the two asymptotically stable minima. During this relaxation, the potential difference $\Delta V$ will be converted into the motion of the state. The regions left and right of the local maximum are basins of attraction for two coexisting attractors. (For potentials with more than one independent variable, $V_{1}$ is often a saddle point rather than a local maximum.)

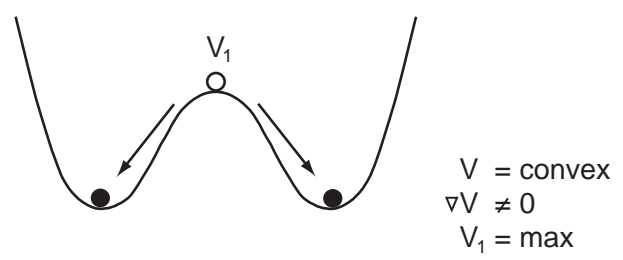

Figure 2. States in the neighborhood of a critical point $V_{1}$ of a locally convex potential are unstable and relax into adjacent potential minima.

Note that in equilibrium thermodynamics the potential $V$ has the concrete meaning of free energy, its derivative with respect to temperature is entropy, and the second derivative is specific heat. However, under conditions far from thermal equilibrium, which are compulsory for pattern formation in general and the behavior of living systems in particular, the observables of equilibrium thermodynamics are typically not well-defined.

For this reason, Haken ([9], Chapter 6.7,) suggested a generalized terminology using potentials rather than free energy, control parameters rather than temperature, action (output) rather than entropy, 
and efficiency (change of output) rather than specific heat. ${ }^{3}$ The state distribution for a given potential is related to the potential by a maximum information principle. The state dynamics simply follows the gradient of the potential—or of the set of potentials, if there are multiple coexisting attractors.

Note also that the distribution of an ensemble of states at some given time equals the distribution of one state over time if and only if the considered system is ergodic. Living systems typically violate ergodicity [10], so that ensemble average and time average (taken over infinitely long time, i.e., asymptotically) differ. Additional problems arise since the time evolution of most systems in nature is only piecewise stationary or not stationary at all (see [11]).

\section{Stability Properties of Mental States}

Recent decades saw a steady trend to describe the behavior of neural and mental systems as nonlinear dynamical systems. Freeman [12] was the first to introduce corresponding approaches, for other accounts see [7,13-16].

The central idea (cf. [17]) is that internal mental representations, the basic elements of a mental system, play the role of attractors (in the sense of Section 2) for external (sensory) or internal stimuli. The relation between the description of mental systems and the formal theory of dynamical systems can be characterized by three points:

1. The mental system, which may be materially realized by a neural network, is treated as a dynamical system $S$.

2. Mental representations within the mental system are treated as co-existing attractors of $S$ with particular stability properties.

3. Internal or external stimuli within the mental system are treated as initial conditions of $S$, whose temporal evolution leads to an attractor.

In this scenario, mental processes, which map a stimulus onto a mental representation, are illustrated by the motion of a state in a potential (or a potential landscape). The form of the potential reflects the stability properties governing this motion. The states themselves and their associated observables can be conceived in two basically different ways. One can consider either mental states or states of the material/neural system correlated with them-notwithstanding the fact that these correlations are often poorly understood.

Our subsequent discussion focuses on the former option, so that the notion of "state" refers to a mental state $\phi$ of a mental system (which can be conscious or unconscious). The state space mostly remains unspecified insofar as there is, to the best of our knowledge, no (canonical) formalization of mental observables. Furthermore, there is no analytic form of the dynamics of states $\phi(t)$ because no corresponding equations of motion are known. The significance of the potential $V$, including its operationalization, remains undefined as well. In particular, we resist the temptation to interpret $V$ as "mental energy" or "psychic energy" as long as such terms are not developed beyond the status of metaphors.

\subsection{Mental Representations and Stable Categorial States}

For conceptual clarity, it is not only helpful but compulsory to distinguish mental representations from mental states. While mental representations are modeled by potentials $V$ constructed over a state space, mental states $\phi$ moving across a set of representations (a potential hypersurface) can aquire different kinds and degrees of stability. The dynamics of states and of representations typically operate at different time scales: state changes (e.g., thinking) are expected to be fast as compared to changes of representations (e.g., learning).

3 Although Friston's "free energy principle" [2] does not follow this terminological move, it should be charitably understood in this generalized way. 
A state $\phi$ can be located anywhere on a potential hypersurface, which defines whether $\phi$ is stable or unstable at its location. This means that mental representations (potentials, attractors) provide constraints for the motion of a mental state $\phi(t)$ as a function of time. If a mental state is located in the minimum of a potential (i.e., on an attractor), the corresponding mental representation is "activated" or "actualized". This means that both its intentional and phenomenal content are subject to experience.

If a mental state is located in a particular mental representation, it is (asymptotically) stable with respect to perturbations (compare Figure 1b). The degree of stability can be quantified by Ljapunov exponents. Representations with shallow potential minima stabilize mental states less than those with deep potential minima. Accordingly, less or more "effort" is necessary for a mental state to "leave" a particular representation. If this happens, the corresponding mental representation may be considered as "deactivated", but as such it stays intact for future "reactivation".

Effectively deactivating a mental representation means that the state $\phi(t)$ has to be changed to such an extent that it leaves not only the attractor but also its basin of attraction. In a landscape of multiple coexisting attractors, $\phi(t)$ will then move towards another attractor to eventually arrive there. A corresponding dynamics of $\phi(t)$ obviously exceeds the conceptual repertoire of a situation with a stable state in the presence of one single representation (as depicted in Figure 1b).

However, not only states can change over time, $\phi=\phi(t)$, but also potential surfaces $V=V(t)$ can evolve. Not only can the dynamics of a state activate or deactivate an already existing mental representation, but new representations can be generated or existing ones can be altered. New representations are created when the mental system generates new potentials; old representations are changed when the corresponding potentials are deformed.

It should be pointed out again that the illustration in Figure $1 \mathrm{~b}$ refers to an especially simple special case, a cartoon picture as it were. Mental representations are not limited to fixed point attractors or limit cycles (periodic processes), but in general correspond to chaotic attractors [18]. Any such asymptotically stable state that activates a mental representation with intentional and phenomenal content, is called a categorial state after Gebser ([19], p. 285; see also [3]):

Every categorial system is an idealized ordering schema by which actual phenomena are fixed and absolutized; as such it is a three-dimensional framework with a static and spatial character. Such categorial systems are able to deal with the world only within a three-dimensional and conceptual world-view.

In the representational account of mental systems due to Metzinger [1], stable categorial states and mental representations (potentials, attractors) that they may activate are the key players. The model of reality that a subject develops consists of two major components: a self-model and a world-model. These models become successively differentiated during an individual's development so that they can map all internal and external experiences that this individual learns to model in order to successfully cope with her or his environment.

By being "fixed and absolutized" à la Gebser, the established repertoire of stable categories that a mental system has at its disposal executes a "censor-like" or "filtering" function [20]. ${ }^{4}$ This function enables clear-cut yes/no attributions of experiences to representations, but it fails to explain how representations are formed and changed, and how states move across representations. If a mental system would be comprised of nothing else than categorial states, this would exclude a manifold of experiences that we will address in the next sections.

4 Note that this kind of filtering is at variance with the notion of the "filter" metaphor often ascribed to the 19th century psychologist Myers. In her knowledgable study of Myers' work on the mind-body problem, Kelly [21] finds that, rather than being due to Myers himself, the metaphor was launched and popularized by Bergson, Broad, Huxley, and others to address the interface between the ordinary conscious experience of an individual and what Myers called the "subliminal self", the tertium quid beyond mind and body. 


\subsection{Marginal Stability of Pre- or Non-Categorial States}

Marginal stability of a state $\phi$ as illustrated in Figure 1a characterizes the limiting case in which $V$ is constant anywhere in the neighborhood of $\phi$. This case can be interpreted as an "unbounded" state which cannot activate any representation, simply because there is none. Consequently, no intentional content will be experienced. Nevertheless, such states can be experienced phenomenally, and it feels somehow if they are. In this respect, one can speak of a non-categorial or pre-categorial state in the absence of representations. Smallest perturbations, which are neither damped nor amplified $(\lambda=0)$, will cause $\phi$ to change.

Metzinger ([1], Chaps. 4 and 7) discusses such states in considerable detail, mostly in relation to his main target: the representation of the self, the phenomenal self-model. It is Metzinger's thesis that the self-model can be modified or lost in altered or pathological states of consciousness. As an example, he refers to the phenomenon of "oceanic" self-dissolution, which he relates to "mystical" states. The associated loss of the phenomenal self does not only presume a change of perspective but rather abolishes experiences of intentional content as they occur under ordinary circumstances.

However, phenomenal experience does not (necessarily) get lost together with a lost self-model. Here, a "window of presence" is activated as a limiting case of state consciousness, a minimal or basic kind of phenomenal content capable of becoming conscious. Because no representations are involved in this limiting case, the experience is unstructured, a uniform atemporal nowness without the perspective of a first person. ${ }^{5}$ Since this scenario abstains from any categories, it refers to a non-categorial state.

Non-categorial states in the absence of particular representations are examples for a subclass of experiences with non-conceptual content, a topic that has gained momentum since it was introduced into the philosophy of mind by Evans [22]. A comprehensive review of this development is due to Bérmúdez and Cahen [23]. For a discussion of non-conceptual content in connection with non-categorial states see [24]. Non-categorial states without conceptual content may be an interesting theme in relation to the spectrum of mental autonomies [25], but this would go beyond the scope of this article.

\subsection{Mental Instabilities and Acategorial States}

Insofar as mental states are asymptotically stable, a transition from one to another is possible only via an unstable intermediate state. Figure 2 illustrates this situation. To effect the transition, $\phi(t)$ has to overcome the potential barrier $\Delta V$. The higher the barrier and the steeper the gradient of the potential, the smaller is the probability of a spontaneous transition and the longer the dwell time of $\phi(t)$ in the potential.

William James ([26], p. 243) gave a succinct description of this interplay of stable and unstable states across a potential landscape:

When the rate [of change of a subjective state] is slow, we are aware of the object of our thought in a comparatively restful and stable way. When rapid, we are aware of a passage, a relation, a transition from it, or between it and something else.... Let us call the resting-places the "substantive parts", and the places of flight the "transitive parts", of the stream of thought. It then appears that the main end of our thinking is at all times the attainment of some other subjective part than the one from which we have just been dislodged. And we may say that the main use of the transitive parts is to lead from one substantive conclusion to another.

A particularly illustrative and frequently studied class of such transitions is the perception of bi- or multistable stimuli (see, e.g., [27] for an overview). In James's terminology, the process leading

5 The experience is subjective only in the weak sense that it is based on an internal model of reality ([1], p. 559). There is just a ground of reality-the unstructured fundament of the manifold of structured aspects of reality. 
from one to another "substantive" state corresponds to a temporal sequence of "transitive" states. The unstable point at $V_{1}$ in the bistable example in Figure 2 is a "transitive" state distinguished by a local maximum of $V$. James ([26], p. 243) continued:

\begin{abstract}
Now it is very difficult, introspectively, to see the transitive parts as what they really are. If they are but flights to a conclusion, stopping them to look at them before the conclusion is reached is really annihilating them. Whilst if we wait till the conclusion be reached, it so exceeds them in vigor and stability that it quite eclipses and swallows them up in its glare. Let anyone try to cut a thought across in the middle and get a look at its section, and he will see how difficult the introspective observation of the transitive acts is.... The results of this introspective difficulty are baleful. If to hold fast and observe the transitive parts of thought's stream be so hard, then the great blunder to which all schools are liable must be the failure to register them, and the undue emphasizing of the more substantive parts of the stream.
\end{abstract}

This is a clear appeal to the effect that the study of unstable, transitive states deserves more attention, but it is also clear why this is hard to do. It is a matter of time scales. While stable states are (usually) adopted long enough to become a subject of conscious experience, this is (usually) not the case for unstable states. Gebser ([19], p. 308) addressed transitive states at a local maximum of $V$ with his concept of acategoriality and emphasized their temporal, dynamical aspect:

Something with a temporal character cannot be spatially fixed. It cannot be fixed or prescribed in any form, and if we attempt to do so we change it by measurement into a spatial quantity and rob it of its true character. This is a clear indication that the qualities of time which are today pressing toward awareness cannot be expressed in mere categorial systems.

Gebser's "unfixable temporal qualities" can-in a more prosaic way-be related to the evasive, transient behavior of complex systems around instabilities. Even if there were known equations of motion to govern the system's behavior on its attractor, its trajectory in the vicinity of instabilities could not be predicted by them.

Acategorial states offer a second option to look at so-called "mystical" experiences, in addition to what Metzinger focuses on. The ground of consciousness cannot only be experienced in the non-categorial minimal kind of representing activity, but also in an acategorial mode. This is possible if some representational ground is present simultaneously with the presence of representations-however, not as an unstructured background as in non-categorial states. Representations remain intact as such and coexist with an awareness of the representational ground. In this case, the self would not be experienced as dissolved but as a sustained yet non-activated representation.

Acategorial states enable an experience of both representational ground and particular representations simultaneously. This is possible if a mental state is not located within a representation (category) but betweem them. In such "in-between states" different representations can be experienced as possibilities without being individually actualized. Dynamically speaking, the continuous representational ground of a mental system can flash up between individual actualized representations and become consciously and phenomenally accessible.

\title{
4. Exceptional Experiences
}

If a mental representation is activated by a categorial mental state, this amounts to an experience of both its intentional and phenomenal content. Such experiences are called ordinary if they are consistent with typical models of reality that individuals develop to cope with their socio-cultural and physical environment. In modern societies, basic elements of such models are established epistemological concepts (such as cause-and-effect relations) and scientific principles and laws (such as gravitation). Experiences inconsistent with those basic elements or deviating from them are considered exceptional (see [28] for more details).

A suitable and viable classification of exceptional experiences (EEs) has been proposed by Fach [29] on the basis of a few key postulates of Metzinger's theory of mental representations. According to 
Metzinger, human mental systems produce a phenomenal reality-model (PRM) that comprises all mental states consciously experienced at a given time. Two fundamental components or base representations within this global model of reality are the self-model and the world-model. ${ }^{6}$ The distinction between the two may seem to resemble the Cartesian distinction of res cogitans and res extensa, but there is a decisive difference: While Descartes' dualism is ontologically conceived, Metzinger's distinction is explicitly epistemic.

The PRM is the overall representation without which no representation in conscious experience can manifest itself-it is the carrier of the experiencing subject and the world that it experiences. Subject-centered experiences condense into cognitive models of reality that subjects create, develop, modify during their lifetime. Since the formation of these models is always embedded in the fundamental structure of the PRM, experiences and the resulting reality concepts are determined by the duality of self-model and world-model.

The world-model contains representations of states of the material world, including an individual's own bodily features. The referents of these representations are observationally accessible and provide intersubjective knowledge, sometimes called "objective" or "third-person" knowledge. The self-model contains representations of internal mental states, such as sensations, cognitions, volitions, affects, motivations, inner images. As a rule, experiences of these states are possible only by an individual itself-they are "subjective" and based on "first-person" accounts.

World-model and self-model are often experienced as strongly correlated. For instance, the bodily organs or limbs, representations in an individual's world-model, and bodily sensations, representations in an individual's self-model, are usually experienced in strong mutual relationship. Nevertheless, we can distinguish self and world. Mental states induced by external sensory stimuli differ from states generated by internal processing. Individuals are usually capable of distinguishing their inner images, affects and fantasies from their perception of physical events in their world-model.

EEs typically appear as deviations ${ }^{7}$ in an individual's reality model. This entails a classification of EEs based on two pairs of phenomena [29]. One pair refers to deviating experiences within the subject's self model and world model, while the other refers to the way in which elements of those models are merged or separated above or below ordinary ("baseline") correlations. The resulting four classes of EE will be characterized in the following.

\subsection{EEs as Deviations from Ordinary Reality-Models}

Whether or not mental representations in the PRM are perceived as exceptional in the sense of a deviation from the ordinary depends on individual and collective knowledge and assumptions about self, world and their interrelation. Even if people are able to integrate EEs in their reality model (their worldview), an EE will continue to deviate from ordinary experiences. The deviation can be attributed either directly from the subject's first-person perspective or through the social environment's third-person perspective on the experience. Deviations from ordinary reality models can occur in both the self-model and the world-model. We refer to deviations in the world-model as external phenomena and to deviations in the self-model as internal phenomena.

1. External phenomena are experienced in the world-model. They include visual, auditory, tactile, olfactory, and kinetic phenomena, the impression of invisible but present agents, inexplicable

6 This definition is based on a footnote in Metzinger ([30], p. 62): “Repräsentationale Gesamtzustände werden durch die Gesamtheit aller zu einem gegebenen Zeitpunkt bewusst erlebten mentalen Modelle gebildet. Sie bestehen aus dem aktuellen Weltmodell und dem aktuellen Selbstmodell und bilden das gegenwärtige Realitätsmodell des Systems."

7 Such deviations are often referred to as "anomalies", or else "psychic" or "psi" experiences. We prefer the notion of a deviation because the theoretical approach taken here entails particular basic classes of such deviations that can be systematically distinguished. This renders the term "anomalies" (in the sense of singular unsystematic occurrences) to be at least arguable-if not inappropriate. 
bodily changes, phenomena concerning audio or visual recordings or the location or structure of physical objects.

2. Internal phenomena are experienced in the self-model. They include somatic sensations, unusual moods and feelings, thought insertion, inner voices, and intriguing inner images. As in class (1), the affected individual is convinced that familiar explanations are suspended, and the experiences appear egodystonic.

In addition to classes (1) and (2), which are constructed as opposites, i.e., independent, there are also deviations in the correlations between self-model and world-model. Strictly speaking, they are experienced as relational between internal (self-model) and external (world-model), so their characterization is based on internal and external phenomena though not contained in (1) or (2). Key to their proper understanding is the relation between (1) and (2), and how it is constituted.

3. Coincidence phenomena refer to experiences of relations between self-model and world-model that are not founded on regular senses or bodily functions, but instead exhibit connections between ordinarily disconnected elements of self-model and world-model. Typically, these excess correlations are assumed to be non-causal, often experienced as a salient meaningful link between mental and physical events. ${ }^{8}$

4. Dissociation phenomena are manifested by disconnections of ordinarily connected elements of self-model and world-model. For instance, individuals are not in full control of their bodies, or experience autonomous behavior not deliberately set into action. Sleep paralysis, out-of-body experiences ${ }^{9}$ and various forms of automatized behavior are among the most frequent phenomena in this class, which is characterized by deficit correlations.

Atmanspacher and Fach [33] showed how this classification can be drawn from a specific kind of dual-aspect framework of thinking originally proposed by Pauli and Jung: the Pauli-Jung conjecture, some details of which we will address in Section 5. Moreover, they indicated how the idea of correlations beyond (excess) and below (deficit) a baseline can be sensibly incorporated into the picture. This baseline is neither universally prescribed, nor is it rigid-it is likely to depend on cultural contexts as well as on previous experiences of the concerned individual. This should be testable for intersubject distributions over individuals and intrasubject distributions over time, respectively. In addition, the intensity of an experience can be regarded as a measure of its distance from the baseline, i.e., of its degree of exceptionality or the extent to which it deviates. See Sections 5.2 and 5.3 for more detailed discussion.

\subsection{Empirical Material}

\subsubsection{Documentation of Counseling Cases}

In order to check how relevant the classification outlined in the preceding section is, it must ideally be compared with empirical data. Since the experiences are denoted as exceptional, one might assume that their frequency of occurrence is low in an interindividual sense and that, as a result, not much empirical material be available. However, this is in fact not the case. Several studies have estimated frequencies of about $50 \%$ for populations in Western countries, and higher than that within other cultural contexts (see, e.g., [34] and further references therein). The reason why EEs are denoted exceptional is that their intraindividual frequency is small as compared to ordinary, non-deviant mental experiences.

8 Meaningful coincidences such as "synchronicities" à la Jung [31] are examples, including extrasensory perception and related phenomena.

9 A challenging discussion of out-of-body experiences based on the concept of phenomenal models of intentionality relations is due to Metzinger [32]. We will come back to this concept in Section 5.2. 
First tests of the classification proposed above were peformed at the Institute for Frontier Areas of Psychology and Mental Health (IGPP) at Freiburg, ${ }^{10}$ where a comprehensive corpus of reports of EEs has been collected since 1996 with a documentation system especially developed to capture EEs in great detail. Principal component analyses and variable-oriented cluster analyses yielded a distribution of reported phenomena as shown in Figure 3 (for more details, see [28,29]). A recent study with a second sample of IGPP clients from 2007 to 2014 as well as the evaluation of the total sample of 2381 reports of counseling cases from 1996 to 2014 confirmed these results [35].

These studies allow us to conclude that the classification of EE patterns into four basic classes of phenomena is on solid empirical ground. Figure 3 shows that classes (1) and (2) are uniquely mapped by the empirical material, whereas classes (3) and (4) split into two subclasses each. Those subclasses can be delineated by a slight dominance of external or internal features in the overly disconnected or overly connected psychophysical relationships that define them.

\section{coincidence phenomena}

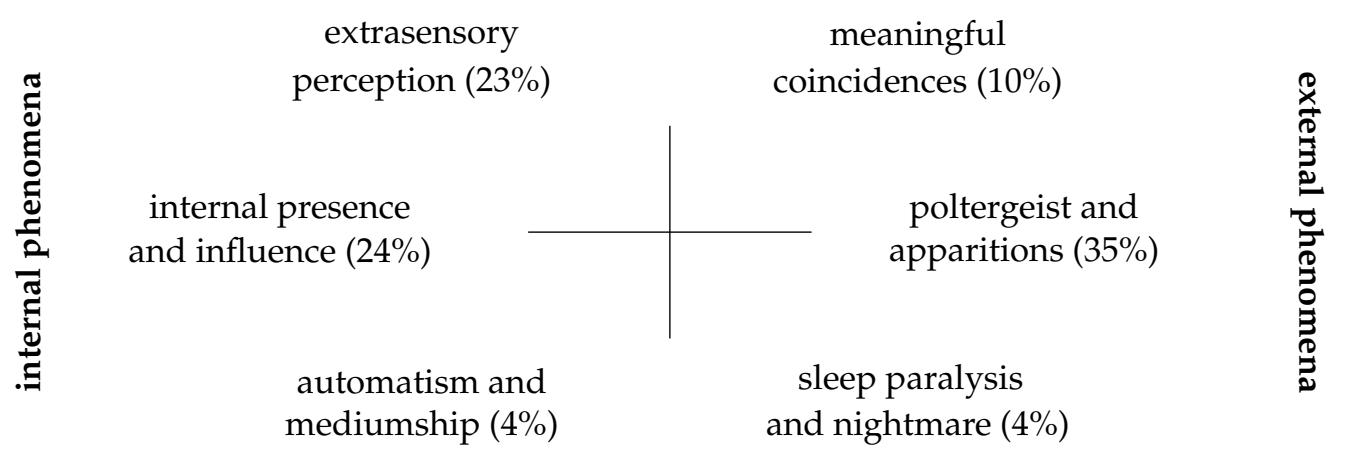

\section{dissociation phenomena}

Figure 3. Empirically assessed experience patterns (based on a total of 2356 reports of counseling cases at IGPP that could be assigned to the six EE-patterns) support the classification derived from the conceptual scheme in Sections 4.1 and 4.2. Internal and external phenomena are deviations in the self model and world model, respectively. Coincidence phenomena show connections (excess correlations) of ordinarily disconnected elements of self and world model. Dissociation phenomena show disconections (deficit correlations) of ordinarily connected elements of self and world model.

More than $95 \%$ of all EE reports discussed so far invoke categorial states, i.e., are about experiences that are made when a categorial state is located in a mental representation. Classes (1) and (2) are clearly categorial since they concern elements of either self- or world-model. In contrast, EE reports in classes (3) and (4) refer to elements of both self- and world-model, but their experiential substance is relational rather than reified. In this sense, classes (3) and (4) are conceptually different from (1) and (2): the particular correlation they express, be it excess or deficit, is typically experienced as meaningful (or salient). Ultimately, it is the relation that constitutes the EEs, not the related categories. This is a key point for an interesting link to Metzinger's discussion of intentionality that we will pick up in Sections 5.2 and 5.3 .

Note that all EE reports were assessed with respect to their phenomenology only, not concerning their veridicality or even psychopathology. ${ }^{11}$ A premature and unjustified identification of EEs with

10 The acronym IGPP derives from the German "Institut für Grenzgebiete der Psychologie und Psychohygiene".

11 The issue of how to assess the veridicality (or "truthfulness") of EEs is delicate, since it bears critically on assumptions about ontology. From a physicalist perspective, the veridicality of an EE requires that it must not contradict the laws of physics. If it 
mental disorders is avoided by the phenomenological approach. In line with previously published results $[36,37]$, health assessments indicate that the majority of clients is mentally healthy. An estimated $1 / 3$ to $1 / 2$ of EE clients seeking advice were either previously or at the time of counseling in psychotherapeutic or psychiatric treatment [35]. Even if $46 \%$ of the clients show signs of mental disorders, the symptoms are often subclinical or not directly associated with EEs.

\subsubsection{Comparative Questionnaire Studies}

In addition to the analysis of case reports from 1996 until 2014 at IGPP, a number of studies with different data pools, diverse target variables, and varied analysis techniques have been conducted with the revised "Questionnaire for Assessing the Phenomenology of Exceptional Experiences" (PAGE-R) [38]. ${ }^{12}$ The PAGE-R was created to collect data systematically by self-assessments of subjects reporting EEs. While the documentation by IGPP counseling staff (Section 4.2.1) records EEs relevant during the ongoing counseling process, the PAGE-R registers EEs over the entire lifetime of subjects. In four sections of eight items each for the basic phenomena classes and a five-level Likert scale, the frequencies of particular phenomena are assessed. Time frames, states of consciousness, external circumstances, subjective evaluation, and personal importance of EEs are also retrieved. The PAGE-R items are formulated to record localizations of and relations between mental representations in the subjects' PRM.

As part of a research project with the Psychiatric University Clinic Zurich, an online survey of Swiss general population $(n=1352)$ with the PAGE-R was carried out in 2011. A comparison with IGPP clients $(n=176)$, questioned in a follow-up survey in 2012, resulted in different frequencies and intensities [28]: IGPP clients rated their EEs significantly higher (about 50\%) than subjects from the general population. ${ }^{13}$

Meanwhile, data from six PAGE-R samples in total, collected between 2011 and 2017, are available: ${ }^{14}$

1. an online survey sample of Swiss general population $(n=1352)$ collected in 2011 as part of a research project of the Psychiatric University Clinic Zurich with Collegium Helveticum Zurich [28].

2. a sample $(n=334)$ of students attending lectures by Ott (IGPP) at the University of Giessen from 2011 to 2014, as well as students from Freiburg [40];

3. an online survey sample $(n=148)$ collected by Simmonds-Moore (University of West Georgia) in 2014 on the geographic spread of EE in the United States (the first use of the PAGE-R in English translation);

4. a sample $(n=272)$ with subjects seeking advice at the counseling service of IGPP between 2007 and 2015 completed the PAGE-R in follow-up surveys from 2012 to 2015;

5. an online survey sample $(n=176)$ with subjects with (at least subjective) near-death experiences was analyzed by Nahm and Weibel with scientific support by IGPP in 2015 to examine EE as aftereffects of near-death experiences;

6. a sample with meditators $(n=59)$ of between 3 and 46 years practice to study effects of meditation [41].

does, the EE will likely be ditched as a psychopathological impairment, a hallucination that, however, will still be regarded veridical from a phenomenological perspective. From a dual-aspect perspective, there may be EEs that are subject to neither physics nor psychology, e.g., relational experiences connecting the physical with the mental. To dismiss the veridicality of such EEs as hallucinations because they do not follow the laws of physics would be an obvious category mistake.

12 The acronym derives from the German "Phänomenologie Ausser-Gewöhnlicher Erfahrungen-Revidiert". It is a proprietary product of IGPP and can be made available for scientific purposes upon requested permission.

13 In this context, let us point out that Wyss [39] showed for the first time how subjects tending to coincidence experiences can be delineated from subjects tending toward other phenomena classes based on psychophysical laboratory experiments.

14 In-depth descriptions of these samples, their analyses, and results can be found in [35]. Publications by the indicated investigators who collected the data for samples 3, 4 and 5 are in progress. 
Factor analyses, cluster analyses, item analyses and scale analyses were used to examine the reliability and validity of PAGE-R in the German-language samples 1, 2, 4, and 5 [35]. The theory-compliant extraction of four factors turned out to be the most robust and best-generalizable model in all these samples not only from an ensemble point of view. Rather, they support a universal latent order in the manifestation of EEs for each individual, in agreement with the theoretical dual-aspect framework indicated in Section 4.1. We may assume that the four-factor model is appropriate for populations of individuals with a PRM exhibiting an intact dichotomy of self-model and world-model.

Figure 4 shows the frequencies of phenomena of the four basic classes of Figure 3 for the six samples mentioned above. The relative distribution of frequencies over the basic classes constituting EEs is comparable for all samples. The US sample 3 indicates that this distribution even holds for non-European populations. As one might expect, frequencies are lowest for samples from general population (samples 1 and 2) and highest for meditators (sample 6) and subjects reporting near-death experiences (sample 5). For the latter, it is unclear whether increased EE frequency favors near-death experiences or is rather a consequence of them.

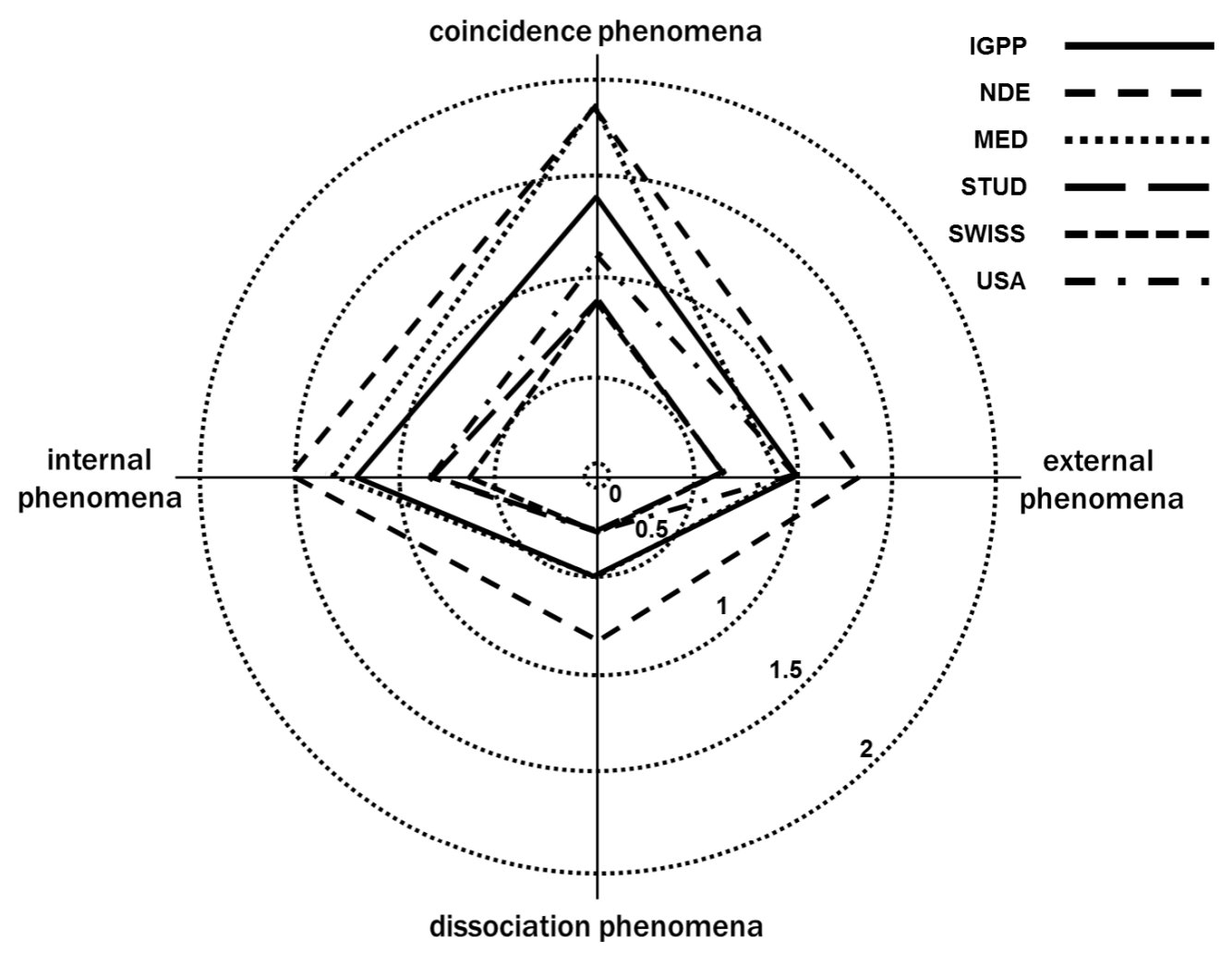

Figure 4. Frequencies of the four basic classes of phenomena from the six samples mentioned in the text. Dotted circles represent frequency levels as assessed individually on a scale from 0 (never) to 4 (very often). IGPP—sample 4, NDE—sample 5, MED—sample 6, STUD-sample 2, SWISS—-sample 1, USA-sample 3 .

The fact that all EE patterns occur not only in clients seeking help, but also, although less often, in the general population shows that EEs are widespread and continuously distributed in their intensity and frequency. In all samples, EEs occur predominantly in the waking state and mostly spontaneously. Mental techniques, drugs, contact with occultism and healers do not play major roles.

A final note of caution: based on a sample with $n=206$ of Swiss general population, Unterrassner et al. [42,43] found a factor-analytic solution with three factors and use these factors as scales for measuring "psychotic-like experiences" by "odd beliefs", "dissociative anomalous 
perceptions" and "hallucinatory anomalous perceptions". As shown in [35], this analysis suffers from two technical shortcomings: (1) a sample size of only 200 persons is too small for reliable conclusions if the general population with its low EE-scores is to be examined and (2) an interpretation of factors as scales is not straightforward and, in this case, does not satisfy the criteria of test theoretical standards.

More serious at the conceptual level is that Unterrassner et al. [42,43] confused the phenomenological paradigm underlying the PAGE-R with their psychiatrically motivated approach. Mistaking experiences for "odd beliefs" or other psychiatric symptoms means to seriously misunderstand the psychopathologically neutral intention that is an essential ingredient of the PAGE-R. From a more general point of view, it is illegitimate to identify EEs with beliefs anyway: believing in EEs does not require having ever experienced one, and experiencing an EE does not presuppose believing in them.

\subsection{Categorial EEs versus Non-Categorial or Acategorial EEs}

Categorial EEs as deviations in the PRM are to be distinguished from experiences of an altered PRM. This can occur in basically two ways: either the differentiation of self-model and world-model regressively fades away in a non-categorical EE or it is progressively transcended in an acategorial EE $[7,29]$. Only categorial mental states (with their intentional content) have the stability properties necessary to be subject to ordinary experience. Non-categorial and acategorial states are either marginally stable or outright unstable and (usually) do not permit the experience of a stable state with intentional content in the usual sense.

EEs based on non-categorial or acategorial states are likely to be more radical than those based on categorial states. Non-categorial states are states in which categorial distinctions are dissolved or not yet established; they form a representational ground as discussed in Section 3.2. Acategorial states are unstable and usually evasive states between existing representations. It remains to be studied whether, and how, they may be stabilized, i.e., prevented from relaxing into neighboring representations, with particular techniques or under particular conditions (see [44] for examples). Other than non-categorial states, typically reported as descending into dark, diffuse and unconscious depths, acategorial states are experienced as light, lucid, and sublime-immanent experiences, as some have called them.

Such experiences sometimes even transcend the distinction between self and world, inside and outside, subject and object. Marshall [45] has an impressive example of a light experience that starts relational, i.e., between inside and outside, and becomes immanent, i.e., transcends that distinction:

I suddenly found myself surrounded, embraced, by a white light, which seemed both to come from within and from without, a very bright light but quite unlike any ordinary physical light...I had the feeling of being "one" with everything and "knowing" all things...I had the sense of this being utter Reality, the real Real, far more real and vivid than the ordinary everyday "reality" of the physical world.

More examples concerning the rich phenomenology of EEs in acategorial states have been described in [7]. Their relevance for the topic of mental states with non-conceptual content [23] has been addressed in [24].

\section{Self-Model and World-Model as Dual Aspects?}

\subsection{Models as Aspects}

Emphasizing that the notions of self and world ought to be understood as models, namely self-model and world-model, entails that they must be models of something. This something could be an ontic reality as such, if reality were not already understood as a model, namely the reality-model, in the representational account that Metzinger advances. This leaves us with the question of how his metaphysical position actually looks. Neither in Being No One [1] nor in the precis of it [46] does he spend much time or space to concisely unveil this position to his readers. Given the very detailed and comprehensive, clarifying and insightful definitions, explanations, and consequences that Metzinger formulates in his texts, this appears to be a noteworthy point. 
Often Metzinger talks about brains or organisms as if they were physical entities, and this may have led some commentators (such as [47], pp. 80-100) to assume that he defends a physicalist stance. However, brains and organisms are part of the world, so if the world is actually to be conceived as a model, then this includes brains and organisms. Thus, alluding to brains or organisms as "real" physical carriers of models means to treat them both as physical entities and as their models at the same time. This injects a poignant ambiguity into an assumed physicalist ontology for Metzinger's framework.

Of course, there is nothing wrong in principle if one talks about both an object domain of reality and models of this domain. However, it is well known that an assumed ontic reality is everything else than in one-to-one correspondence with models that physicists, biologists, or brain scientists have developed and utilize. This is obvious from the fact that different viable models of the same object domain can be incompatible or even logically exclusive, for instance deterministic and stochastic approaches, or computational and phenomenological approaches to "the brain". In view of these complications, it is quite difficult to delineate an object domain and its model(s) in a way that is clear-cut enough to avoid confusion between the two.

We think that this confusion can be most elegantly avoided by turning to a powerful alternative to physicalism that receives increasing attention in recent years: dual-aspect thinking. Now it has to be understood right away that dual-aspect thinking is not a monolithic building of thought, but comes in a number of variants to be sketched below. A basic feature of dual-aspect accounts is that the mental and the physical (self and world, roughly) are not conceived as ontic entities but rather as aspects, or perspectives. The question then is, very similar to models in Metzinger's parlance, aspects or perspectives of what are they?

With respect to this question, dual-aspect thinkers bite the bullet and admit that present-day science has almost nothing to say about the base reality, except that it should be psychophysically neutral, i.e., neither mental nor physical. (It is certainly not the reality of fundamental physical particles or fields.) Nevertheless, there are several speculative ideas of how to characterize this psychophysically neutral in more detail. We will return to this below in Section 5.5. For the discussion right now, let us just assume the psychophysically neutral as a tertium quid from which its mental and physical aspects (self and world, roughly) arise or emerge.

There are basically two conceptions in which this emergence can be addressed. ${ }^{15}$ In one of them, psychophysically neutral elementary entities are composed of sets of such entities and, depending on the composition, these sets acquire mental or physical properties. Major historic proponents of this compositional scheme are Mach, James, Avenarius, and Russell. In the literature, this is often referred to as "neutral monism" [49,50] A neo-Russellian version of neutral monism proposed by Chalmers [51] has been quite influential, both in the philosophy of mind and in cognitive neuroscience (cf. the work of Tononi and his group [52]).

The other base conception is close to Spinoza's way of thinking, where the psychophysically neutral does not consist of elementary entities waiting to be composed, but is conceived as one overarching whole that is to be decomposed. In contrast to the atomistic picture of compositional dual-aspect monism, the holistic picture of the decompositional variant is strongly reminiscent of the fundamental insight of holism and nonlocality in quantum physics. Quantum systems are wholes that can be decomposed in infinitely many complementary ways, very much like how Spinoza's idea of the divine has been interpreted.

Inspired by quantum holism, modern decompositional dual-aspect thinking has been mainly proposed by philosophically inclined physicists in the 20th century, starting with Bohm and Pauli (together with the psychologist C.G. Jung). Subsequent work along the same lines has been due to

15 A compact account of 20th century examples of dual-aspect thinking can be found in [48], including commentaries by Horst, Seager, and Silberstein. 
d'Espagnat, Primas, and our own proposals refining the Pauli-Jung conjecture [4]. ${ }^{16}$ A key difference between compositional and decompositional accounts is that the mental and the physical are reducible to neutral elements if these are the basis for composition, but they are irreducible (in the standard understanding of reduction) to a neutral whole if this is the basis for decomposition.

Another important point is that decomposing a whole necessarily implies correlations between the emerging parts, while composition does not necessarily give rise to correlations between different sets of composed elements. In this way, decompositional dual-aspect monism has been highlighted as the one philosophical framework that explains mental-physical correlations most elegantly and parsimoniously. The price to be paid is that the metaphysics of a psychophysically neutral whole is largely undeveloped and leaves much work to be done.

\subsection{Intentionality}

At a panel discussion some time ago, one of us (HA) had an exchange with Godehard Brüntrup (GB), a Munich based philosopher, that was directed at the nature and our understanding of psychophysical correlations. Quoted from memory, it went like this:

GB: You know, we have sensory capacities for vision, the eyes, for hearing, the ears, and so on. However unfortunately, we don't have a sense for psychophysical correlations!

HA: Wait a minute! I'm not sure about this—-though the latter may differ fundamentally from the former. How about the sense of meaning?

GB: What do you mean, meaning? Intentionality?

HA: Yes-if you want to use this phil-of-mind term. Anyway, it's about the experience of something that, roughly speaking, connects a mental representation with what it represents. Meaning in this sense is a relational experience, and this is what makes it fundamentally different from the ordinary senses.

GB: I can see where you're going ... I have to think about it.

Intentionality is the technical term for the reference relation that may be colloquially called a relation of meaning. The notion of the intentional content of a mental representation, in addition to its phenomenal content, addresses what the representation refers to. Metzinger ([46], Figures 1 and 2) illustrates the way in which the traditional way to conceive intentionality ("good old-fashioned intentionality") according to Brentano deviates from his conception. While intentionality is traditionally conceived as a relation between a mental representation and what it represents in the world out there, he conceives intentionality as a relation between a mental representation and the representation of the world out there.

In other words, Metzinger does precisely what we were asking for in Section 5.1: he talks about models of the world rather than the world itself. The brain, or the organism, or the universe as a whole, all need to be addressed in terms of models. This does not exclude that there may be some reality out there, but it would be illegitimate to identify it with the various ways in which it can be modeled. Thus, Metzinger's focus in [46] is clearly on intentionality as a relation between self-model and world-model, not between self-model and world itself.

Insofar as the phenomenal experience of intentionality is relational, it cannot be the phenomenal content of one of the related representations. Now, in order to account for the actual experience of intentionality (as meaning!) in a representational way, Metzinger postulates a phenomenal model of the intentionality relation (PMIR) as a meta-representation transcending the level of those first-order representations that are related by intentionality. The PMIR allows him to address the experience of meaning in terms of the phenomenal content of a meta-representation.

From the dual-aspect perspective on exceptional experiences outlined in Section 4.1 (see also Figure 3), the classes of coincidence and dissociation phenomena in EEs can be straightforwardly linked

16 In recent years, an increasing number of affective and cognitive neuroscientists have emphasized the potential of dual-aspect approaches, such as Damasio, Friston, Hobson, Panksepp, Solms, Velmans, and others. 
to PMIRs. EEs are experiences of relations between elements of the self-model and the world-model of an experiencing subject. The phenomenal expression of those experiences is encoded in the meta-representation of the PMIR as its phenomenal content. The degree of exceptionality of an EE depends on its deviation from the baseline of a default intentionality that subjects employ under ordinary, everyday circumstances.

\title{
5.3. Transparency and Opacity
}

In addition to relational EEs as PMIRs, Metzinger's approach even gives us a clue to characterize the deviation of relational EEs from the baseline by a special property of representations in general and PMIRs in particular. Representations can be transparent or opaque ([53], p. 7): ${ }^{17}$

\begin{abstract}
Transparency is a property of conscious representations, namely that they are not experienced as representations. Therefore, the subject of experience has the feeling of being in direct and immediate contact with their content. Transparent conscious representations create the phenomenology of naive realism. An opaque phenomenal representation is one that is experienced as a representation, for example in pseudo-hallucinations or lucid dreams. Importantly, a transparent self-model creates the phenomenology of identification ([1], Section 3). There exists a graded spectrum between transparency and opacity, determining the variable phenomenology of "mind-independence" or "realness".
\end{abstract}

What we denote as a baseline of relational experiences, a default intentionality that subjects employ under ordinary circumstances, is hardly ever explicitly experienced, and even less so as meaningful. For instance, no one attributes meaningful experiences to the correlation between their mental states and their neural states. The corresponding baseline, thus, perfectly illustrates a relational experience whose PMIR is characterized by complete transparency. Note that this example differs from a transparent first-order representation of, say, an apple, which appears to us as an object of reality out there, in the sense of naive realism. However, even if we realize that the apple actually is an element of our world-model, a transparent PMIR of this situation would mean that we do not attribute any explicit meaning to seeing the apple.

There is one key feature in mind-body relations whose PMIR is typically transparent in many instances: the experience of mineness, or ownership. This is the impression that a particular mental representation of a bodily state (or action) of a subject is related to the bodily state (or action) of that same subject- "my" bodily state (or action). In dissociation phenomena, mineness and ownership can be severely impaired, i.e., ordinarily connected elements of the self-model and the world-model of the subject become disconnected. In this situation, we speak of deficit correlations, below the baseline. Out-of-body-experiences are an illustrative case in point: The mental state of the subject represents its body, as an element of the world-model, in a way that appears disconnected from the representing self-model.

In such a case, the subject realizes that the connection of its body-model to its self-model deviates from the ordinary, so the PMIR of this connection is not transparent but achieves (gradual) opacity. And insofar as such a case is experienced as highly meaningful (especially if it is the first time it happens), it deviates considerably from the baseline. This leads us to the hypothesis that the extent to which an EE deviates from the baseline of relational experiences be a measure of the opacity of its corresponding PMIR. Furthermore, this hypothesis is equally sensible if coincidence phenomena are at stake, where excess rather than deficit correlations are responsible for the deviation from the baseline.

We should add that the concept of a baseline sounds more rigid than we think it should be thought of. Although our empirical material provides evidence that we have a pretty stable baseline on an average over thousands of subjects (in Western cultural contexts), individual variation has to be

17 This important distinction goes back to Moore [54] and, later, Harman [55], who used the term "transparent" roughly in Metzinger's sense, while van Gulick [56] applies it differently. An informative discussion of a number of readings of transparency is due to Stoljar [57]. 
expected. For instance, it will be likely that subjects who learn how to induce EEs of a certain kind will shift their baseline toward the corresponding class of phenomena. As a result, an EE that was outstanding when it was experienced first will lose intensity if it is volitionally reproduced. More work on EEs and their link to opaque and transparent PMIRs is in progress.

\subsection{Self and World Again}

Metzinger's main thesis in Being No One "is that no such things as selves exist in the world: Nobody ever was or had a self. All that ever existed were conscious self-models that could not be recognized as models" ([1], p. 1)—because of their transparency. While Rudder Baker's move [47] is to reinstantiate experiencing selves as ontologically existing subjects, we think with Metzinger that the notion of the self in the sense of a "mediator" of first-person experience should not be given ontological significance. However, as pointed out above, we would insist that this equally applies to the physical world insofar as it is a world of modeled and observed, hence epistemic, physical objects. While Baker wants to pull the self into ontology, we would argue that even the physical world is basically epistemic —namely, conceived as a world-model à la Metzinger —and should be pushed out of ontology.

It is central to most dual-aspect approaches that the mental and the physical are considered as epistemic insofar as their experiencable and observable structures and dynamics are by definition subject to knowledge. However, over and above this dual-aspect thinking posits a knowledge-independent ontic reality without the mind-matter or subject-object split. As mentioned In Section 5.1, decompositional dual-aspect monism is inspired by the wholeness or nonlocality of quantum theory, which illustrates why observed objects need to be regarded as epistemic. Physical nonlocality is empirically demonstrated by measuring correlations between subsystems that are generated by measurement itself, so that the original wholeness of the not-yet-measured (ontic, as it were) system is destroyed.

On the psychological side, Jung ([58], supplement) has argued that this view on quantum measurement has an analogy in the transition from unconscious to conscious states: the wholeness of mental states at the archetypal level of the unconscious is destroyed when they are transformed into selected conscious contents. Ultimately, the idea is that the most comprehensive level of mental unconsciousness and the most comprehensive level of physical wholeness converge-a limit in which there are no distinctions left at all.

The notion of the self, as Metzinger and most other philosophers of mind use the term, is essentially bound to the so-called first-person perspective of "what it is like" to be in a given mental state. An individual which mistakes its self-model as an individual self is bound to a number of interpretive flaws that Metzinger's account discloses. It may be interesting to see that the role of Metzinger's self-model resembles the Jungian notion of an ego that fails to realize its status as being purely epistemic. However, Jung also has the notion of an impersonal, non-individual "self" characterizing an overarching archetypal structure within the psychophysically neutral reality, whose fragmented projection in an individual's conscious psyche functions as an ego, i.e., Metzinger's self-model. ${ }^{18}$

\subsection{From One to Many, and Back}

In the decompositional variety of dual-aspect monism, the ultimate psychophysically neutral reality is one whole, a whole with no internal structure, no distinctions, no parts, no time, no space. In the Pauli-Jung conjecture, this is called the unus mundus, the one world, from which all diversity arises. It's a modern creation myth, if you wish. In addition, since there are no distinctions, there is no

18 For commonalities (and differences) of this conception with Eastern philosophies such as versions of Buddhism and Hinduism see the readable collection of essays by Siderits et al. [59]. Notably, some of the authors discuss concrete relations to Metzinger's work. 
discursive epistemic access to this reality. It is "unspeakable", or "ineffable". Alter and Nagasawa [50] discuss a number of options to conceive this unspeakable-which they refer to as "inscrutable".

A formal way to approach the undividedness of this reality is in terms of symmetry principles. A symmetry in the technical sense is an invariance under transformations. When we say that a circle is invariant under the transformation of a rotation, we mean that it does not change its appearance, no matter which angle it is rotated about. In this sense, the circle is symmetric with respect to rotation. Casually speaking, symmetry is about "change without change".

Quantum field theory is a success story of the power of symmetries in physics: The way we understand the fundamental physical forces today is a series of symmetry breakdowns, leading to more and more refined concepts of matter and energy. Now, the unus mundus is not physical of course, it is psychophysically neutral, and its mental and physical aspects are undefined because of their lacking distinction. However the mathematical principle of a symmetry transcends its applications in physics, so symmetry offers itself as an interesting candidate to address other domains of reality as well. ${ }^{19}$

Increasing refinements of an unus mundus due to successive distinctions are expressible as symmetry breakings, such as the mental versus the physical, subject versus object, inside versus outside, self versus world. In the Pauli-Jung conjecture, there is a primordial split-up of the unus mundus into a collective mental unconscious (transcending individual subjects) and a holistic physical world (transcending observable objects), raw forms of the mental and the physical that are yet to be differentiated. More refinements lead to the full-blown mental domain with its multiplicity of consciously experienced states (a self-model) and a full-blown physical domain with its multiplicity of observable and manipulable phenomena (a world-model). Other variants of dual-aspect thinking propose different accounts [48].

The unspeakability and inscrutability of an unus mundus block cognitive discursive access, and relational experiences are impossible if there are no distinct mental and physical states to be related. However, the unus mundus need not exclude experience altogether. As indicated in Section 4.3, there are conceivable modes of experience that do not require well-defined distinct states and relations between them. Rather, they may be targeted directly at the undifferentiated ground from which distinct states emerge. Such immanent experiences may be understood as non-categorial (or even acategorial) states without conceptual, or categorial, content [24].

Immanent experiences are experiences reversing the direction "from one to many" back to one, from the refined concepts of our familiar consciousness back toward the limit of total undividedness. The literature on mystical experiences contains numerous examples of this move, as for instance displayed and discussed in [45]. Mystical experiences are hard to communicate in conventional language and logic, and corresponding attempts often result in paradoxical formulations such as Plotinus' identity in difference or Nicolas of Cusa's coincidentia oppositorum (see also [60]). They express experiences of the one undifferentiated ground that itself does not enter into the contents of representations arising from it.

Are immanent experiences meaningful? If meaning is a relation explicated through an epistemic split, as in intentionality, then they are not. However, insofar as immanence points to the source of those representations that are related by intentionality, experiences of immanence may refer directly to this source and unveil the implicit origin of a meaning that is yet to be explicated. However careful: without the epistemic split of subject and object, such experiences cannot be subjective in the usual sense any more! William James [61] speculated about them with his notion of "pure experience", others have called them experiences of "pure presence" or "non-dual awareness".

19 Symmetry principles do not exhaust the range of possibilities to address the psychophysically neutral formally. Other options that one might think of are non-commutative structures, non-Boolean logic, or even mathematical fields as general as category theory. 
Author Contributions: Conceptualization, H.A.; investigation, H.A. and W.F.; data curation, W.F.; original draft preparation, H.A.; review and editing, H.A. and W.F.

Funding: This research received no external funding.

Acknowledgments: This article is dedicated to Thomas Metziger on the occasion of his 60th birthday. His innovative, careful and ground-breaking body of work on the model of the self, with all its intricacies, has been a source of inspiration for many, including the authors. We thank Ulrich Ott, Michael Nahm, Stefan Schmidt, Alexander Siller, Christine Simmonds-Moore, Adrian Weibel and Marc Wittmann for making their data available to us prior to publication. In addition, we are grateful for helpful comments of the referees of this article.

Conflicts of Interest: The authors declare that the work presented in this article was carried out without any personal, professional or financial relationships that could potentially be construed as a conflict of interest.

\section{References}

1. Metzinger, T. Being No One. The Self-Model Theory of Subjectivity; MIT Press: Cambridge, MA, USA, 2003.

2. Friston, K. A free energy principle for biological systems. Entropy 2012, 14, 2100-2121.

3. Atmanspacher H. Categoreal and acategoreal representation of knowledge. Cogn. Syst. 1992, 3, $259-288$.

4. Atmanspacher, H.; Fuchs, C.A. (Eds.) The Pauli-Jung Conjecture; Imprint Academic: Exeter, UK, 2014

5. Guckenheimer, J.; Holmes, P. Nonlinear Oscillations, Dynamical Systems, and Bifurcations of Vector Fields; Springer: Berlin, Germany, 1983.

6. Leven, R.W.; Koch, B.-P.; Pompe, B. Chaos in Dissipativen Systemen; Akademie: Berlin, Germany, 1994.

7. Atmanspacher, H.; Fach, W. Acategoriality as mental instability. J. Mind Behav. 2005, 26, 181-206.

8. Alligood K.T.; Sauer T.D.; Yorke, J.A. Chaos. An Introduction to Dynamical Systems; Springer: Berlin, Germany, 1996.

9. Haken, H. Synergetics. An Introduction; Springer: Berlin, Germany, 1977.

10. Molenaar, P.C. On the implications of the classical ergodic theorems: Analysis of developmental processes has to focus on intra-individual variation. Dev. Psychobiol. 2008, 50, 60-69. [CrossRef] [PubMed]

11. Priestley, M.B. Non-linear and Non-stationary Time Series Analysis; Academic Press: New York, NY, USA, 1988.

12. Freeman W.J. Nonliner dynamics of pleocortex manifested in the olfactory EEG. Biol. Cybern. 1979, 35, 21-34. [CrossRef] [PubMed]

13. Beer R.D. Dynamical approaches to cognitive science. Trends Cogn. Sci. 2000, 4, 91-99. [CrossRef]

14. Fell, J. Identifying neural correlates of consciousness: The state space approach. Conscious. Cogn. 2004, 13, 709-729. [CrossRef]

15. Kaneko, K.; Tsuda, I. Complex Systems: Chaos and Beyond; Springer: Berlin, Germany, 2001.

16. van Gelder T. The dynamical hypothesis in cognitive science. Behav. Brain Sci. 1998, 21, 615-661. [CrossRef]

17. Nicolis, J. Chaotic Information Processing; World Scientific: Singapore, 1991.

18. Skarda, C.; Freeman, W. How brains make chaos in order to make sense of the world. Behav. Brain Sci. 1987, 10, 161-173. [CrossRef]

19. Gebser, J. The Ever-Present Origin; Ohio University Press: Columbus, OH, USA, 1986.

20. Emrich, H.M.; Leweke, F.M.; Schneider, U. Systems-theory of psychosis-The relevance of "internal censorship". Pharmacopsychiatry 2006, 39, 52-53. [CrossRef]

21. Kelly, E.W. F.W.H. Myers and the empirical study of the mind-body problem. In Irreducible Mind; Kelly, E.F., Kelly, E.W., Crabtree, A., Grosso, M., Greyson, B., Eds.; Rowman \& Littlefield: Lanham, MD, USA, 2007; pp. 47-115.

22. Evans, G. The Varieties of Reference; Oxford University Press: Oxford, UK, 1982.

23. Bérmúdez, J.; Cahen, A. Nonconceptual mental content. Stanford Encyclopedia of Philosophy; Zalta. E.N., Ed. 2015. Available online: plato.stanford.edu/entries/content-nonconceptual/ (accessed on 31 January 2019).

24. Feil, D.; Atmanspacher, H. Acategorial states in a representational theory of mental processes. J. Conscious. Stud. 2010, 17, 72-104.

25. Metzinger, T. The myth of cognitive agency: Subpersonal thinking as a cyclically recurring loss of mental autonomy. Front. Psychol. 2013, 4, 1-19. [CrossRef]

26. James, W. Principles of Psychology; Dover: New York, NY, USA, 1890.

27. Kruse, P.; Stadler, M. (Eds.) Ambiguity in Mind and Nature; Springer: Berlin, Germany, 1995.

28. Fach, W.; Atmanspacher, H.; Landolt, K.; Wyss, T.; Rössler, W. A comparative study of exceptional experiences of clients seeking advice and of subjects in an ordinary population. Front. Psychol. 2013, 4, 65. [CrossRef] 
29. Fach, W. Phenomenological aspects of complementarity and entanglement in exceptional human experiences. Axiomathes 2011, 21, 233-247. [CrossRef]

30. Metzinger, T. Subjekt und Selbstmodell; Mentis: Paderborn, Germany, 1999.

31. Jung, C.G. Synchronicity-An acausal connecting principle. In Collected Works Vol. VIII; Princeton University Press: Princeton, NJ, USA, 1952.

32. Metzinger, T. Out-of-body experiences as the origin of the concept of a soul. Mind Matter 2005, 3, 57-74.

33. Atmanspacher, H.; Fach, W. A structural-phenomenological typology of mind-matter correlations. J. Anal. Psychol. 2013, 58, 219-244. [CrossRef]

34. Schmied-Knittel, I.; Schetsche, M.T. Everyday miracles: Results of a representative survey in Germany. Mind and Matter 2013, 10, 169-184.

35. Fach, W. Das Spektrum des Aussergewöhnlichen. Konzeptionelle Ansätze, empirisch-phänomenologische Untersuchungen und plananalytische Fallstudien zur mentalen Repräsentation bei aussergewöhnlichen Erfahrungen. Ph.D. Thesis, University of Bern, Bern, Switzerland, 2019.

36. Belz-Merk, M.; Fach, W. Beratung und Hilfe für Menschen mit aussergewöhnlichen Erfahrungen. Psychotherapie-Psychosomatik-Medizinische Psychologie 2005, 55, 256-265. [CrossRef]

37. Belz, M.; Fach, W. Theoretical reflections on counseling and therapy for individuals reporting ExE. In Perspectives of Clinical Parapsychology; Kramer, W.H., Bauer, E., Hövelmann, G.H., Eds.; Stichting Het Johan Borgman Fonds: Bunnik, The Netherlands, 2012; pp. 168-189.

38. Fach, W. Lehmann, und Abteilung Beratung und Information IGPP Revidierter Fragebogen zur Erfassung der Phänomenologie aussergewöhnlicher Erfahrungen (PAGE-R). Institut für Grenzgebiete der Psychologie und Psychohygiene (IGPP), Freiburg, Germany, Unpublished questionnaire, 2011.

39. Wyss, T. Behavioral, Physiological and Subjective Aspects of Exceptional Experiences. Ph.D. Thesis, ETH Zurich, Zurich, Switzerland, 2016.

40. Siller, A.; Ambach, W.; Vaitl, D. Investigating expectation effects using multiple physiological measures. Front. Psychol. 2015, 6, 1553. [CrossRef]

41. Schmidt, S.; Jo, H.-G.; Wittmann, M.; Ambach, W.; Kübel, S. Remote meditation support-A multimodal distant intention experiment. Explore 2018, in press. doi.org/10.1016/j.explore.2018.12.002 [CrossRef]

42. Unterrassner, L.; Wyss, T.; Wotruba, D.; Ajdacic-Gross, V.; Haker, H.; Rössler, W. Psychotic-like experiences at the healthy end of the psychosis continuum. Front. Psychol. 2017, 8, 775 [CrossRef]

43. Unterrassner, L.; Wyss, T.; Wotruba, D.; Haker, H.; Rössler, W. The intricate relationship between psychotic-like experiences and associated subclinical symptoms in healthy individuals. Front. Psychol. 2017, 8, 1537. [CrossRef]

44. Atmanspacher, H.; Filk, T.; Scheingraber, H. The significance of causally coupled, stable neuronal assemblies for the psychological time arrow. In Endophysics, Time, Quantum and the Subjective; Buccheri, R., Elitzur, A.C., Saniga, M., Eds.; World Scientific: Singapore, 2005; pp. 149-162.

45. Marshall, P. Mystical Encounters with the Natural World; Oxford University Press: Oxford, UK, 2005.

46. Metzinger, T. Precis: Being No One. Psyche 2005, 11, 1-35.

47. Rudder Baker, L. Naturalism and the First-Person Perspective; Oxford University Press: Oxford, UK, 2013.

48. Atmanspacher, H. 20th century variants of dual-aspect thinking. Mind Matter 2014, 12, 245-288.

49. Stubenberg, L. Neutral monism. In Stanford Encyclopedia of Philosophy; Zalta, E.N., Ed. 2016. Available online: plato.stanford.edu/entries/neutral-monism/ (accessed on 31 January 2019).

50. Alter, T.; Nagasawa, Y. What is Russellian monism? J. Conscious. Stud. 2012, 19, 67-95.

51. Chalmers, D. The Conscious Mind; Oxford Univesity Press: Oxford, UK, 1996.

52. Tononi, G. Integrated information theory. Scholarpedia 2015, 10, 4164. Available online: dx.doi.org/10.4249/ scholarpedia.4164 (accessed on 31 January 2019).

53. Metzinger, T. The problem of mental action-Predictive control without sensory sheets. In Philosophy and Predictive Processing; Metzinger, T., Wiese, W., Eds.; MIND Group: Frankfurt am Main, Germany, 2017. Available online: predictive-mind.net/papers (accessed on 31 January 2019).

54. Moore, G.E. The refutation of idealism. Mind 1903, 12, 433-453. [CrossRef]

55. Harman, G. The intrinsic qualia of experience. Philos. Perspect. 1990, 4, 31-52. [CrossRef]

56. van Gulick, R. A functionalist plea for self-consciousness. Philos. Rev. 1988, 97, 149-181. [CrossRef]

57. Stoljar, D. The argument from diaphanousness. Can. J. Philos. 2004, 34, 341-390. [CrossRef] 
58. Jung, C.G. On the nature of the psyche. In Collected Works Vol. VIII, Princeton University Press: Princeton, NJ, USA, 1954.

59. Siderits, M.; Thompson, E.; Zahavi, D. (Eds.) Self, No Self? Perspectives from Analytical, Phenomenological, and Indian Traditions; Oxford University Press: Oxford, UK, 2010.

60. Bagger, M. The Uses of Paradox; Columbia University Press: New York, NY, USA, 2007

61. James, W. Does “consciousness" exist? J. Philos. Psychol. Sci. Methods 1904, 1, 477-491. [CrossRef]

(C) 2019 by the authors. Licensee MDPI, Basel, Switzerland. This article is an open access article distributed under the terms and conditions of the Creative Commons Attribution (CC BY) license (http:/ / creativecommons.org/licenses/by/4.0/). 